\title{
PCI effects in Argon 2p double Auger decay probed by multielectron coincidence methods
}

\author{
S Sheinerman ${ }^{1,2,3}$, P Lablanquie ${ }^{1,2}$, F Penent ${ }^{1,2}$, Y Hikosaka , T $^{4}$ \\ Kaneyasu $^{5}$, E Shigemasa ${ }^{5}$ and K Ito ${ }^{6}$ \\ ${ }^{1}$ LCP-MR, Université Pierre et Marie Curie (UPMC), 11 rue P et M Curie, 75231 \\ Paris Cedex 05, France \\ ${ }^{2}$ CNRS, LCPMR(UMR 7614), 11 rue Pierre et Marie Curie, 75231 Paris Cedex 05, \\ France \\ ${ }^{3}$ Department of Physics, St. Petersburg State Maritime Technical University, 198262 \\ St. Petersburg, Russia \\ ${ }^{4}$ Department of Environmental Science, Niigata University, Niigata 950-2181, Japan \\ ${ }^{5}$ UVSOR Facility, Institute for Molecular Science, Okazaki 444-8585, Japan \\ ${ }^{6}$ Photon Factory, Institute of Materials Structure Science, Oho, Tsukuba 305-0801, \\ Japan
}

PACS numbers: $32.70 . J z, 32.80 . \mathrm{Hd}$

\begin{abstract}
Electron correlations in three electron emission associated with innershell photoionization are investigated for the first time through a combined theoretical and experimental approach. Namely, different kinds of Post Collisional Interactions (PCI) occurring in the decay of Argon 2p holes by emission of two Auger electrons are isolated experimentally with a powerful coincidence technique and are modeled with an eikonal approach. It is shown that PCI distortion of the photoelectron line shape is stronger than in the single Auger paths and depends critically on the process: in direct Double Auger decays where two Auger electrons are emitted simultaneously, direct interaction of the $2 p$ photoelectron with the two Auger electrons is demonstrated; in cascade Double Auger processes, the PCI distortion depends only slightly on the last electron emitted in the cascade. This perturbation depends on and reveals the lifetime of the intermediate $\mathrm{Ar}^{2+}$ state.
\end{abstract}




\section{Introduction}

An inner atomic vacancy created by photoionization can decay by emission of radiation or by Auger decay. For L shells, the Auger decay is generally more probable and can occur with emission of one electron (single Auger decay) or of a few electrons (multiple Auger decay). The probability of multiple Auger decay is usually lower than the probability of single Auger decay but can be high enough to allow experimental observations (see e.g. Aberg 1975). The first evidence of a double Auger (DA) process was reported by Carlson and Krause $(1965,1966)$. Recently, progress in coincidence measurements opened the path to a deeper search of the double Auger decays (Penent et al 2005). DA implies that the filling of the inner shell vacancy by an outer electron can cause the ejection of two electrons. This process is in its turn divided into the direct double Auger decay (DDA) when the two electrons are emitted simultaneously and cascade double Auger decay (CDA) when the electrons emission occurs in two steps through the creation and decay of an intermediate quasi-stationary state. Viefhaus et al 2004 were the first to demonstrate directly the existence of the DDA path.

Recently, coincidence measurements based on the use of magnetic bottle time of flight spectrometers (Eland 2009) have opened the possibility to obtain the complete state selected triple photoionization (TPI) continua over a wide range of kinetic energies (Hikosaka et al 2009). The application of this method to the multi-electron decays of an Ar 2 p inner shell reveals the following (Lablanquie et al 2007, Palaudoux et al 2008, Hikosaka et al 2009, 2009A): i) One can distinguish the electrons emitted due to the decay of the $2 p_{3 / 2}$ subshell or of the $2 p_{1 / 2}$ one. ii) The observed electron lines can be unambiguously filtered according to the final state (e.g. configuration ...3 $3 s^{2} 3 p^{3}$ with the different terms $\left({ }^{4} S,{ }^{2} D,{ }^{2} P\right)$.) iii) One can separate the electron lines with respect to the decay process of the $2 \mathrm{p}$ innershell: emission of one, two and up to three Auger electrons. iv) The position and shapes of the photoelectron lines which are associated with the DA decay differ for the DDA and CDA processes. v) The line shapes ascribed to the DA processes are strongly distorted by post collision interaction (PCI) effects.

$\mathrm{PCI}$ is known as a special kind of electron correlation associated with the interaction between the charged particles of a resonant process, through the creation and decay of an intermediate quasi stationary state. In the case of inner shell photoionization, PCI reduces to the interaction of the emitted photoelectron with the Auger electrons and with the ion field which varies during the Auger decay. PCI in single Auger decay following inner shell photoionization is quite well documented both experimentally and theoretically (see for example the reviews of Kuchiev and Sheinerman 1989, Schmidt 1992).

PCI in the DA processes has been much less studied. There are a number of experimental investigations on the PCI influence on the threshold photoelectron yield in the vicinity of inner vacancies: $\operatorname{Kr} 1 s^{-1}, \operatorname{Kr} 2 p^{-1}$, Kr $3 p^{-1}$, Ar $1 s^{-1}$, Ar $2 p^{-1}$, Xe $4 p^{-1}$, Xe $4 d^{-1}$ (Hayaishi et al 1988,1990,1994, Kjeldsen et al 1996, Hayaishi et al 1999,2000,2002, Matsui et al 2002). These experiments present coincidences between threshold electrons, 
which are the more affected by PCI effect, and the residual ions. PCI in DA processes was also studied after $4 \mathrm{~d}$ ionization in Xenon by the method of threshold electron / Auger electron coincidences (Lablanquie et al 2001, 2002). Finally only a few studies presented a theoretical analysis of PCI associated with few Auger electron decay: a classical model to describe the release of threshold electrons in the DA process (Kjeldsen et al 1996), a classical model of cascade Auger decay (Koike 1994), and a quantum mechanical eikonal approach to the DDA and CDA processes (Sheinerman 1994, 1998).

In this study, we reveal and investigate the PCI effects in DA processes, using Ar $2 \mathrm{p}$ innershell ionization as an example. For the first time we present a systematic investigation of photoelectron spectra measured in coincidence with two Auger electrons. The PCI distortion of the photoelectron and Auger lines is shown to be important for DDA and CDA processes. Our measurements are supported by calculations based on an eikonal approach to PCI. The results of these calculations are in good agreement with the experimental data thus demonstrating the adequacy of the theoretical model to the phenomenon considered. The analysis of the experimental and theoretical results shows that the photoelectron line shape depends both on the variation of the ionic field and on the interaction with the Auger electrons. Moreover, since the line shapes for DDA and CDA processes are slightly different, their analysis allows us to extract properties of the associatted DA decays.

The paper is organized as follows. Section 2 presents a brief description of our experimental method and shows 2 dimensional maps of the coincidences for double Auger decays. Section 3 presents the theoretical approach for calculation of the cross section of the DA processes. In section 4 we present the results of our measurements with calculation and the analysis of these data. The atomic system of units $|e|=m_{e}=\hbar=1$ is used throughout.

\section{Experiment}

Two different experimental setups have been used here: a magnetic bottle time of flight analyzer for powerful coincidence detection and a very high resolution hemispherical analyzer for the spectroscopic characterization of the $\mathrm{Ar}^{2+}$ states involved in the cascade double Auger decay.

The non coincident high resolution spectra were obtained on the brand new undulator beamline BL6U at the UVSOR storage ring in the Institute for Molecular Science in Okazaki, Japan. BL6U is equipped with a variable included angle grazing incidence monochromator with a varied line spacing plane grating, without entrance slit. We used a high performance hemispherical electron energy analyzer MBS-A1, developed by the MB Scientific AB company. It is equipped with a gas cell and a CCD camera and reaches a theoretical $1.3 \mathrm{meV}$ (FWHM) resolution when operated with a pass energy of $2 \mathrm{eV}$ and a slit opening of $0.2 \mathrm{~mm}$. The energy scale was calibrated with the strongest Ar 2p DA cascade peak (Lablanquie et al 2007), set at $2.19 \pm 0.01 \mathrm{eV}$. We checked carefully this value with the magnetic bottle analyzer, by measuring simultaneously $\mathrm{He}$ 
photoelectron lines, in order to avoid possible shifts due to contact potentials. Slow drift of the peak positions occurred with time and were minimized by short scans of less than a few minutes.

The main part of the results were acquired with a magnetic bottle time of flight analyzer. This kind of apparatus has been extensively described before (Eland et al 2003, Penent et al 2005, Hikosaka et al 2006, Eland 2009) and will only be briefly introduced here. Ionization occurs close $(\sim 1 \mathrm{~mm})$ to a strong $(\sim 0.5 \mathrm{~T})$ permanent magnet in a zone where the strong divergent magnetic field creates a magnetic mirror. Almost all released electrons are guided along the magnetic field lines through a long $(2.5 \mathrm{~m})$ solenoid $\left(\sim 10^{-3} \mathrm{~T}\right)$ to the detector. A multi-hit time-to-digital converter then records the arrival times of each electron with respect to the light bunch and stores them in on-line computers for further analysis. Time to energy calibration was performed by measuring photolines associated with $\mathrm{He}^{+}(\mathrm{N}=1)$ and $\mathrm{He}^{+}(\mathrm{N}=2)$ formation at known photon energies. The experiments were performed at the Photon Factory and used light from the undulator beam line BL-16B (Shigemasa et al 1998). The photon bandwidth was set at $30 \mathrm{meV}$. Single bunch operation of the storage ring provided 200 ps-wide light pulses every $624 \mathrm{~ns}$. It follows that time of flight of isolated electrons could only be measured modulo the 624 ns inter light pulse period. It was however not needed to use a mechanical chopper (Ito et al 2009) to increase this period in this favourable case: it is possible to retrieve absolute electron time of flights in coincidence events where a $2 \mathrm{p}$ photoelectron and all associated Auger electrons are recorded, because one of the Auger electrons is then necessarily a fast one, with a flight time shorter than 624 ns. Typical count rate in coincidence experiments was $3000 \mathrm{~Hz}$, which allows us to neglect random coincidences between electrons produced by subsequent light pulses.

The key point for the success of the present experiment is its ability to define precisely the conditions in which PCI is observed, thanks to the coincidence techniques. Fig $1 \mathrm{a}$ ) and $1 \mathrm{~b}$ ) demonstrate first the ability to resolve the final states reached respectively in double and triple ionization events. The histogram of the sum of the kinetic energy of two electrons detected in coincidence in Fig 1 a) reveals the $\mathrm{Ar}^{2+}$ final state. Most of the events correspond to the creation and Auger decay of a $2 p_{1 / 2}$ or a $2 p_{3 / 2}$ innershell hole, along with a minor valence double ionization contribution. Similarly, the final $\mathrm{Ar}^{3+}$ states populated by triple ionization are obtained in Fig. 1 b) through the histogram of the sum of the kinetic energies of three electrons detected in coincidence, and they originate mainly from the decay of $2 p$ holes by emission of two Auger electrons. The resolution, slightly improved compared to our previous study (Lablanquie et al 2007), allows us to distinguish but not isolate fully the $\operatorname{Ar}^{3+}\left(3 p^{-3}\right)^{2 S+1} L$ final states. A clear separation between direct and cascade Double Auger decays is not always possible. For instance, the two processes overlap heavily in the case of the $4 \mathrm{~d}$ decay in Xenon (Penent et al 2005), but the 2p decay in Argon offers a favorable system, where distinction is possible (Viefhaus et al 2004, Lablanquie et al 2007). This is demonstrated in Fig 2, which presents a 2 dimensional coincidence map of the $2 \mathrm{p}$ DA decay measured at the same $\omega=251.63 \mathrm{eV}$ photon energy as for Fig 1 a) and b). Only events where 
3 electrons were detected in coincidence are considered here. A filter on the sum $S$ of the energy of the three electrons ensures that a final $\mathrm{Ar}^{3+}\left(3 p^{-3}\right)$ state is reached. Fig 2 a) represents coincidence counts as a function of the kinetic energy of two electrons of the triplet where intensity is coded in color on a log scale. The kinetic energy of the third electron of the triplet is naturally deduced from the relation $\omega=S+E_{B}\left(\mathrm{Ar}^{3+}\right)$ where $E_{B}\left(\mathrm{Ar}^{3+}\right)$ is the binding energy of the final $\operatorname{Ar}^{3+}\left(3 p^{-3}\right)$ state. One observes in Fig 2 a) two structured vertical bands which correspond to the detection of a $2 p_{1 / 2}$ photoelectron (at around $0.8 \mathrm{eV}$ ) or a $2 p_{3 / 2}$ one (at around $3 \mathrm{eV}$ ). Fig $2 \mathrm{~b}$ ) on the right side gives the projection of the 2 dimensional map onto the ordinate axis. It represents the histogram for the energy of one of the three electrons emitted in triple ionization events. One can isolate clearly from Fig 2 the direct and cascade DA processes: peaks correspond to cascade processes superimposed on a weak structureless contribution that extends vertically over all electron energies and is associated with the direct DA path. We selected in this study two cascade processes characterized by the emission of a $8.5 \mathrm{eV}$ electron for the first one, and or of a $2.19 \mathrm{eV}$ electron for the second. As for the DDA path, we selected also two zones, with emission of an Auger electron in the intervals $(30-70) \mathrm{eV}$ and $(4.9-5.1) \mathrm{eV}$. The experimental coincidence spectra presented in Fig 3,5 and 6 display the $2 p$ photoelectron line shapes associated with the emission of such electrons, they are obtained by projection on the $\mathrm{x}$ axis of the horizontal bands in Fig 2 a) associated with these Auger electrons.

\section{Theoretical approach to PCI effects in DA processes}

Treatment of PCI associated with inner shell photoionization implies taking into account the interaction of the charged particles in the intermediate and final states of the process. It means that the photoelectron interacts first with the ionic field the charge of which varies from +1 to +3 during the DA decay of the inner vacancy. Then this photoelectron interacts with the two Auger electrons which are emitted in the Auger decay. For descriptions of these effects we will use a quantum mechanical eikonal approach which has been used successfully for the case of single Auger decay (Sheinerman et al 2006, Palaudoux et al 2008).

This approach implies that the main contribution to the interaction between the escaping particles occurs at large distances when $R \equiv V_{p h} / \Gamma \gg 1$. Here $V_{p h}$ is the photoelectron velocity and $\Gamma$ is the width of the inner vacancy. In this case we can consider the kinetic energy of the relative motion of the emitted particles to be significantly larger than the potential energy of their interaction. This assumption is valid if the following condition is fulfilled (Kuchiev and Sheinerman 1986): $E_{p h} \gg$ $\Gamma^{2 / 3} \cdot\left(E_{0} / 2\right)^{1 / 3}$ where $E_{0}$ is the atomic unit of energy and $E_{p h}$ is the photoelectron energy. Here, the widths values of $\Gamma\left(2 p_{1 / 2}\right)=\Gamma\left(2 p_{3 / 2}\right)=118 \mathrm{meV}$ (Jurvansuu et al 2001) ensure that this condition is fulfilled rather well for a photon energy in excess over threshold of $E_{p h}>2 \mathrm{eV}$ and quite reasonable for $2 \mathrm{eV}>E_{p h}>0.7 \mathrm{eV}$. Therefore we can use the eikonal approach to analyse our data at least for the energies $E_{p h}>0.7 \mathrm{eV}$. 


\subsection{PCI in direct double Auger decay (DDA)}

This decay occurs when the two Auger electrons are emitted simultaneously. The equation of the reaction has the form:

$$
\begin{aligned}
\omega+\operatorname{Ar} & \rightarrow e_{1}+\operatorname{Ar}^{+*}\left(2 p_{1 / 2,3 / 2}^{-1}\right) \rightarrow \\
& \rightarrow e_{1}+e_{1 A}\left(E_{2}\right)+e_{2 A}\left(E_{3}\right)+\operatorname{Ar}^{3+}\left(\left(3 p^{-3}\right){ }^{4} S,{ }^{2} D,{ }^{2} P\right) .
\end{aligned}
$$

The PCI theory for the DDA processes on the base of the eikonal approach was developed in (Sheinerman 1998). According to this theory the PCI-distorted cross section of DDA process when all three electrons are registered in coincidence is given by :

$$
\begin{aligned}
\frac{d^{4} \sigma}{d E_{2} d E_{3} d \Omega_{3}} & =\int d \Omega_{1} d \Omega_{2} \sigma_{0}\left(\omega, E_{1}^{(0)}, \Omega_{1}\right) \times \\
& \times \frac{\Gamma_{d}\left(E_{2}^{(0)}, E_{3}^{(0)}, \Omega_{2}, \Omega_{3}\right)}{2 \pi\left[\left(\varepsilon_{2}+\varepsilon_{3}\right)^{2}+\Gamma^{2} / 4\right]} \cdot \frac{\pi \xi}{\sinh (\pi \xi)} \exp \left[2 \xi \tan ^{-1} \frac{2\left(\varepsilon_{2}+\varepsilon_{3}\right)}{\Gamma}\right] .
\end{aligned}
$$

Here $E_{1}^{(0)}, E_{2}^{(0)}, E_{3}^{(0)}$ are unshifted values of energies of photoelectron, first and second Auger electrons, which are ejected in the directions characterized respectively by the solid angles $\Omega_{1}, \Omega_{2}, \Omega_{3}$. The energy variables $\varepsilon_{2}, \varepsilon_{3}$ denote the energies of the first and second Auger electrons measured from their unshifted values: $\varepsilon_{2}=E_{2}-E_{2}^{(0)}$, $\varepsilon_{3}=E_{3}-E_{3}^{(0)} . \sigma_{0}$ is the inner shell photoionization cross section and $\Gamma_{d}$ is the partial width of the double Auger decay. It should be noted that the lineshape is determined only by the inner vacancy width $\Gamma$ and by the kinematics of the process which is included in the dimensionless parameter $\xi$ :

$$
\xi=\frac{-2}{V_{1}}+\frac{1}{\left|\mathbf{V}_{1}-\mathbf{V}_{2}\right|}+\frac{1}{\left|\mathbf{V}_{1}-\mathbf{V}_{3}\right|}
$$

with $V_{1}, V_{2}, V_{3}$ being the velocities of the escaping electrons.

\subsection{PCI in cascade double Auger decay (CDA)}

This decay occurs when the two Auger electrons are emitted sequentially. It means that the CDA occurs through the creation and decay of an excited state of the $\mathrm{Ar}^{2+*}$ ion and that the second Auger electron is emitted due to the decay of this intermediate quasistationary $\mathrm{Ar}^{2+*}$ state:

$$
\begin{aligned}
\omega+\operatorname{Ar} & \rightarrow e_{1}+\operatorname{Ar}^{+*}\left(2 p_{1 / 2,3 / 2}^{-1}\right) \rightarrow e_{1}+e_{1 A}\left(E_{2}\right)+\operatorname{Ar}^{2+*} \rightarrow \\
& \rightarrow e_{1}+e_{1 A}\left(E_{2}\right)+e_{2 A}\left(E_{3}\right)+\operatorname{Ar}^{3+}\left(\left(3 p^{-3}\right){ }^{4} S,{ }^{2} D,{ }^{2} P\right) .
\end{aligned}
$$

The theory for the CDA processes based on the eikonal approach was developed in (Sheinerman 1994). According to this theory the PCI distorted cross section of CDA process is given by :

$$
\frac{d^{4} \sigma}{d E_{2} d E_{3} d \Omega_{2}}=\frac{\sigma_{0}\left(\omega, E_{1}^{(0)}\right) \Gamma_{d}^{(1)}\left(E_{2}^{(0)}\right) \Gamma_{d}^{(2)}\left(E_{3}^{(0)}\right)}{4 \pi^{2}\left[\left(E_{j}-E_{i}-E_{2}-E_{3}\right)^{2}+\Gamma_{1}^{2} / 4\right]\left[\left(E_{3}-E_{3}^{(0)}\right)^{2}+\Gamma_{2}^{2} / 4\right]} \times
$$




$$
\begin{gathered}
\int d \Omega_{1} d \Omega_{3}\left|N_{\xi}\right|^{2} \exp \left[2 \xi_{1} \tan ^{-1} \frac{2\left(E_{j}-E_{i}-E_{2}-E_{3}\right)}{\Gamma_{1}}\right] \times \\
\exp \left[-2\left(\xi_{2}+\xi_{3}\right) \tan ^{-1} \frac{2\left(E_{3}-E_{3}^{(0)}\right)}{\Gamma_{2}}\right] \times \\
\left|{ }_{2} F_{1}\left(1+i \xi_{1},-i \xi_{2}, 2+i\left(\xi_{1}+\xi_{3}\right), 1-\frac{E_{3}-E_{3}^{(0)}+i \Gamma_{2} / 2}{E_{2}+E_{3}+E_{i}-E_{j}+i \Gamma_{1} / 2}\right)\right|^{2} .
\end{gathered}
$$

Here ${ }_{2} F_{1}$ is the hypergeometric function symbol and $E_{i}, \Gamma_{1}$ are the energy and the width of the inner vacancy; $\Gamma_{2}$ is the width of the intermediate quasistationary state of the $\mathrm{Ar}^{2+*}$ ion; $\Gamma_{d}^{(1)}$ and $\Gamma_{d}^{(2)}$ are the partial Auger decay widths of the initial and intermediate vacancies; $E_{j}$ is the energy of final ionic state. To simplify we assume the factors $\Gamma_{d}^{(1)}$, $\Gamma_{d}^{(2)}, \sigma_{0}$ do not depend on the direction of the electron ejection.

The dimensionless parameters $\xi_{i}$ are defined as:

$$
\begin{aligned}
& \xi_{1}=\frac{-1}{V_{1}}+\frac{1}{\left|\mathbf{V}_{1}-\mathbf{V}_{2}\right|} ; \\
& \xi_{2}=\frac{-1}{V_{2}}+\frac{1}{\left|\mathbf{V}_{2}-\mathbf{V}_{3}\right|} ; \\
& \xi_{3}=\frac{-1}{V_{1}}+\frac{1}{\left|\mathbf{V}_{1}-\mathbf{V}_{3}\right|}
\end{aligned}
$$

And the normalization factor $\left|N_{\xi}\right|^{2}$ is given by

$$
\begin{aligned}
\left|N_{\xi}\right|^{2}= & \frac{\pi^{2} \xi_{1} \xi_{2}}{\sinh \left(\pi \xi_{1}\right) \sinh \left(\pi \xi_{2}\right)} \cdot \frac{\sinh \left[\pi\left(\xi_{1}+\xi_{2}\right)\right]}{\sinh \left[\pi\left(\xi_{1}+\xi_{2}+\xi_{3}\right)\right]} \times \\
& \frac{\left(\xi_{1}+\xi_{2}+\xi_{3}\right)\left[1+\left(\xi_{1}+\xi_{2}+\xi_{3}\right)^{2}\right]}{\left(\xi_{1}+\xi_{2}\right)\left[1+\left(\xi_{1}+\xi_{2}\right)^{2}\right]}
\end{aligned}
$$

It is important to note that in this case the cross section is determined by both the widths of the initial inner vacancy $\Gamma_{1}$ and of the intermediate doubly charged excited state $\Gamma_{2}$.

The equations (2) and (5) present the cross sections of the processes when all three electrons are registered in coincidence and have precisely define energies $E_{1}, E_{2}, E_{3}$. If we want to describe the real experimental conditions when the photoelectron yield is measured and the energy of one of the Auger electrons, for example $e_{2 A}$, belongs to some definite interval $\Delta E=E_{\text {max }}-E_{\text {min }}$, then these cross sections have to be integrated over the energy $E_{3}$ :

$$
\sigma\left(E_{1}, \Delta E\right)=\int_{E_{\min }}^{E_{\max }} \frac{d^{4} \sigma}{d E_{2} d E_{3} d \Omega_{3}} d E_{3} .
$$

Of course, carrying out of such an integration we have to take into account the energy conservation law:

$$
E_{1}+E_{2}+E_{3}=\omega-E\left(\operatorname{Ar}^{3+}\left(3 p^{-3}\right)\right),
$$


where $\omega$ is the energy of the incident photon.

In the same way we can obtain the line shape of the second Auger electron in the CDA process carrying out an integration of the cross section (5) over the energies of the photoelectron $E_{1}$ :

$$
\sigma\left(E_{3}, \Delta E\right)=\int_{E_{\min }}^{E_{\max }} \frac{d^{4} \sigma}{d E_{2} d E_{3} d \Omega_{3}} d E_{1} .
$$

\section{Results of measurements and calculation}

To analyze the PCI distortion in DA spectra we have selected two energies of the incident photon $\omega=251.63 \mathrm{eV}$ and $\omega=260.63 \mathrm{eV}$ that corresponds to excess energy of $3 \mathrm{eV}$ and $12 \mathrm{eV}$ above the $2 p_{3 / 2}$ threshold.

\subsection{PCI distortion in direct double Auger decay (DDA)}

One of our aims is to investigate the contribution of direct interaction between the photoelectron and Auger electrons to the PCI distortion. Hence we have chosen two different intervals for the Auger electrons which are emitted during the DDA, $\Delta E=30-70 \mathrm{eV}$ and $\Delta E=4.9-5.1 \mathrm{eV}$. As discussed above, analysis of the coincidence spectra shows that the Auger electrons of these energies are ejected purely in DDA processes. The energies of the second Auger electron are in this case approximately $95-135 \mathrm{eV}$ and $160 \mathrm{eV}$ respectively. The corresponding measured and calculated spectra of the coincident photoelectron line shape are presented in the figures $3 \mathrm{a}$ (for $\omega_{1}=251.63 \mathrm{eV}$ ) and $3 \mathrm{~b}$ (for $\omega_{2}=260.63 \mathrm{eV}$ ). In each figure the experimental results are shown as error bars, while calculated data are represented by solid lines. A blue color is used when one of the Auger electron energies belongs to the interval $(\Delta E=30-70 \mathrm{eV})$ while red refers to the second group $(\Delta E=4.9-5.1 \mathrm{eV})$. For comparison, the PCI distortion associated with the decay of $2 p$ holes by emission of a single Auger electron and populating $\operatorname{Ar}^{2+}\left(3 p^{-2}\right)$ final states are represented in black.

First we can see two peaks in the spectra which are associated with the photoelectrons emitted from the $2 p_{1 / 2}$ or $2 p_{3 / 2}$ subshells. The line shapes of these peaks are shifted and distorted by the PCI. The PCI distortion is stronger for smaller energies of the photoelectron in agreement with the general behavior of PCI. It is seen that, generally, the PCI distortion for DDA processes (blue and red curves) is larger than for the single Auger process (black curves) because of the larger variation of the ionic field (on $+2|e|$ units). However it appears that the red and black curves almost coincide in Fig 3b): this is when the photoelectron is faster than one of two Auger electrons (of $5 \mathrm{eV}$ ) emitted in the DDA decay. In this case and in a classical picture, the photoelectron is never overtaken by the slow Auger electron, and always feels a +1 central charge, as in the single Auger decay.

It is clear that the distortion of the photoelectrons lines associated with Auger electrons of $\Delta E=30-70 \mathrm{eV}$ are always stronger than for Auger electrons of 
$\Delta E=4.9-5.1 \mathrm{eV}$. It can be easily understood if we take into account both the interaction of the photoelectron with the ion field and with the slower Auger electron (the interaction of the photoelectron with the fast Auger electron of $>100 \mathrm{eV}$ energy can be neglected). For the photoelectron energies considered here the main contribution comes from the interaction with the ion field. The weaker interaction with the slower Auger electron has an opposite sign and its contribution is larger for the smaller energies of the Auger electron (see the equation (3)). As a result, the difference between the photoelectron line shapes associated with Auger electrons of different energies shows clearly the important role of direct interaction between photo and Auger electrons.

We can note quite a good agreement between the measured and calculated spectra. Note that all calculated and experimental curves are normalized at their maximum. For the comparison we have convolved the calculated curves with Gaussian of FWHM = $50 \mathrm{meV}$ for $\omega_{1}$ and of FWHM $=150 \mathrm{meV}$ for $\omega_{2}$ to model the experimental resolution. We used the experimental ratio of $0.6: 1$ for the intensities of the $2 p_{1 / 2}$ and $2 p_{3 / 2}$ peaks, as extracted from our measurements at $\omega_{2}=260.63 \mathrm{eV}$. Note that this ratio apparently increases at $\omega_{1}=251.63 \mathrm{eV}$ but no attempt was made to adjust its value in the calculation as it does not improve significantly agreement with experiment for photoelectron energies of less than $1 \mathrm{eV}$ (see below). The energy scale of the calculated curves has been shifted by $+140 \mathrm{meV}$ for $\omega_{1}$ and by $+90 \mathrm{meV}$ for $\omega_{2}$ to match experiment. This discrepancy could arise from the uncertainties in electron and photon energy calibration and in the values of the $2 p$ binding energies that we took from the literature (King et al 1977). Disagreement between calculated and measured data for photoelectron energies of less than $1 \mathrm{eV}$ is expected because the validity of the eikonal approach breaks down for small photon excess energies, and our model does not intend to describe this region precisely. However one notes that the trends of the PCI distortion in this region are also correctly described by our model.

As a preliminary conclusion on DDA processes, we can say that: 1) our coincidence method allows us to reveal the notable contribution to PCI from the interaction between the photoelectron and Auger electrons that strongly depends on the Auger electron(s) energies; 2) the eikonal theory for PCI describes adequately the direct double Auger processes.

\subsection{PCI distortion in cascade double Auger decay (CDA)}

For the analysis of PCI effects in CDA processes we have selected the two strongest cascade paths characterized respectively by the emission of Auger electrons of 2.19 and $8.5 \mathrm{eV}$. These electrons correspond to the second Auger electron emitted in the CDA path. More precisely they are associated with excited $\mathrm{Ar}^{2+*}$ intermediate states of $86.31 \mathrm{eV}(\mathrm{A} 1)$ and $96.94 \mathrm{eV}$ (A2) binding energy which decay respectively towards the $\operatorname{Ar}^{+3}\left(3 p^{-3} S\right)$ and $\operatorname{Ar}^{+3}\left(3 p^{-3} 2 P\right)$ final states (Lablanquie et al 2007). 
4.2.1. Characterization of the $\mathrm{Ar}^{2+}$ intermediate state involved in the cascade double Auger decay Figure 4 shows high resolution electron spectra of the second-step Auger electron of the two CDA processes that we have selected. They were obtained with the MBS high resolution spectrometer at a photon energy of $270 \mathrm{eV}$, in order to minimize the PCI distortion of the peaks. Fig 4 a shows the $2.19 \mathrm{eV}$ peak, measured with a 2 $\mathrm{eV}$ pass energy. Analysis of the peak width in a series of similar measurements reveals a lifetime broadening of $3.7 \pm 0.4 \mathrm{meV}$, taking into account the theoretical $1.3 \mathrm{meV}$ resolution of the analyzer and the $2.9 \mathrm{meV}$ Doppler contributions.

Lifetime broadening of the $8.5 \mathrm{eV}$ peak in Fig 4b) is obviously larger. A $20 \mathrm{eV}$ pass energy was used here and is enough to reveal the doublet structure of the peak, associated with the decay of the $\mathrm{A} 2 \mathrm{Ar}^{2+*}$ intermediate state to the $1 / 2$ and $3 / 2$ spin orbit components of the $\operatorname{Ar}^{3+}\left(3 p^{-3}\left({ }^{2} P\right)\right)$ final state. The fit represented in the Fig $\left.4 \mathrm{~b}\right)$ uses the literature value of $22 \mathrm{meV}$ for this splitting (Ralchenko et al 2008) and takes into account the broadening due to the $13 \mathrm{meV}$ theoretical resolution of the analyzer plus the $5.7 \mathrm{meV}$ Doppler contribution. We deduce a $35 \pm 5 \mathrm{meV}$ broadening associated with the lifetime of the $\mathrm{A} 2 \mathrm{Ar}^{2+*}$ intermediate state.

4.2.2. PCI distortion of the photoelectron lineshape in cascade double Auger decay $(C D A)$ Fig 5 and 6 display in red the distortion of the photoelectron lineshape in CDA paths mediated respectively through the $\mathrm{A} 1$ and $\mathrm{A} 2 \mathrm{Ar}^{2+*}$ intermediate states, which emit a 2.19 or $8.5 \mathrm{eV}$ secondary electron upon their decay. For comparison we also give in black and blue the same curves as in Fig 3, to show the PCI distortion in a single Auger decay (black) and in a direct path (blue) where the two emitted Auger electrons are rather fast (around $50 \pm 20 \mathrm{eV}$ and $115 \pm 20 \mathrm{eV}$ ). The same procedure as above has been adopted for the comparison of the calculated and experimental results (convolution and shift of the theoretical curves). The main result is that red and black curves are almost exactly the same: PCI distortion is almost the same in single Auger decays and in cascade Double Auger decays. It is easily understood if we take into account that the interaction between the photo- and the second Auger electron occurs at large distances where this interaction is small and contributes only a little to PCI. The main contribution to PCI comes from the interaction of the photoelectron with the ion field and this interaction is the same in the single Auger and in the cascade Double Auger process before the second Auger electron emission.

However a closer inspection reveals that if the PCI distortion in single and cascade double Auger paths is the same, within experimental resolution, when we consider path A1 and a $2.19 \mathrm{eV}$ Auger electron in Fig 5, it differs slightly for path A2 in Fig 6. More precisely, a slight difference is visible in Fig 6a) when the photoelectron is slower than the $8.5 \mathrm{eV}$ Auger electron emitted in the last step of the A2 cascade. The experiment reveals that the smaller the photoelectron energy, the larger the difference. This observation implies that the photoelectron can can be influenced by a second Auger electron if it has a lower kinetic energy than this Auger electron. But to reveal this effect another condition is required: that the lifetime of the $\mathrm{Ar}^{2+*}$ intermediate state be short enough. 
That is why we detect this effect for the A2 intermediate state of 19 fs lifetime (35 meV lifetime broadening) in Fig 6a) but not for the A1 state of 180 fs lifetime (3.7 meV lifetime broadening) in Fig 5a). (note that these times have to be compared with the lifetime of the $2 \mathrm{p}$ hole itself, which is $5.6 \mathrm{fs}$ as deduced from its $118 \mathrm{meV}$ lifetime broadening (Jurvansuu et al 2001).

The calculations reproduce exactly this behaviour, when introducing the lifetime of the $\mathrm{Ar}^{2+*}$ intermediate. Fig 5 shows that even with a $\Gamma_{2}=15 \mathrm{meV}$ lifetime broadening, no effect is predicted for path A1. Using a $40 \mathrm{meV}$ lifetime broadening for path A2 reproduces correctly the slight difference of PCI distortion for the red curves around 3 $\mathrm{eV}$ in Fig 6a). Agreement is less good for photoelectron energies smaller than $1 \mathrm{eV}$, as expected in this zone where eikonal approach is not valid, but the trend is correctly reproduced.

Finally this effect should be detected also in the kinetic energy distribution of the second Auger electron emitted in the last step of the cascade path. As expected we did not observe, within experimental error bars, any change in the $2.19 \mathrm{eV}$ line shape associated with the decay of A1 state. In the experiment with the high resolution MBS spectrometer, we observed a distortion and an increase of the apparent kinetic energy of the $8.5 \mathrm{eV}$ peak when decreasing the excess energy above the $2 \mathrm{p}$ thresholds. However the coincidence experiments, in spite of their lower resolution $(120 \mathrm{meV})$, reveal more clearly the effect thanks to the ability to isolate contributions from the $2 p_{3 / 2}$ and $2 p_{1 / 2}$ decays. The result is displayed in Fig 7 , where blue, black and red curves correspond respectively to coincidences of the second step Auger line with a

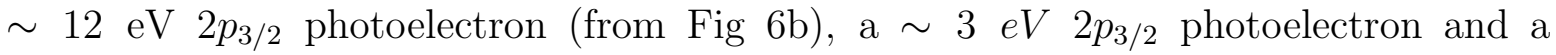
$\sim 0.9 \mathrm{eV} 2 p_{1 / 2}$ photoelectron (from Fig 6a). A shift of the order of a $\sim 100 \mathrm{meV}$ is observed experimentally. The calculations of the second Auger electron distribution (solid curves) was performed by using formula (10) and (5); we use a value $40 \mathrm{meV}$ for the width $\Gamma_{2}$ of the A2 intermediate state. Calculated curves were convolved with a $\mathrm{FWHM}=120 \mathrm{meV}$ Gaussian to take into account the experimental resolution and were all shifted by $-50 \mathrm{meV}$ to match experimental observations. We note a rather good agreement between the measured and calculated curves for photoelectrons energies of $\sim 12 \mathrm{eV}$ and $\sim 3 \mathrm{eV}$.

Hence the PCI distortion of the line shapes of both the photoelectrons and the Auger electrons has been revealed clearly by our investigation of the CDA processes. Note that our approach allows us to distinguish and even select the contribution of different kinds of electron interactions in the total PCI distortion.

\section{Conclusion}

Our paper presents for the first time a detailed investigation of the the PCI interactions between a positive ion and three electrons (1 photoelectron and two Auger electrons) in different conditions. Both our experimental coincidence method and our theoretical eikonal approach allow us to reveal reliably the PCI distortion of the photoelectron 
and the Auger electron line peaks and to show the dependence of this distortion on the energies of the emitted electrons in the DDA and CDA processes. In the case of the CDA decay, the analysis of the second Auger electron line shape allows us to estimate the widths of the intermediate quasistationary $\mathrm{Ar}^{2+*}$ state. The quite good agreement between calculated and measured line shapes show that the eikonal theory of PCI describes adequately the direct and cascade double Auger decays, it also suggests that PCI distortion can be used to judge if a particular Auger electron is released in a direct or cascade Double Auger process. The methods presented here can be extended to the investigation of inner shells in other atomic or molecular systems. A further step will be to test the dependence of the PCI distortions upon the relative emission angles of the photoelectron and Auger electrons. The present success of our model suggests that it should also predict those dependences reliably. On the experimental front, angleresolved 3 electron coincidences are unfortunately beyond the present experimental capabilities.

\section{Acknowledgments}

We are grateful to the Photon Factory staff for the efficient and stable operation of the PF ring. We warmly thank professor John Eland for many discussions and for his constant support. Financial supports from JSPS and CNRS (PICS program no. 3407) are acknowledged. This work was performed with the approval of the Photon Factory Advisory Committee (proposal no. 2004G210).

\section{References}

Electronic address: pascal.lablanquie@upmc.fr; sheinerman@post.ru

Ȧberg T 1975 Atomic Inner-Shell Processes vol I, ed B Crasemann (New York: Academic) p 353

Carlson T A and Krause M O 1965 Phys. Rev. Lett. 14390

- 1966 Phys. Rev. Lett. 171079

Eland J H D, Vieuxmaire O, Kinugawa T, Lablanquie P, Hall R I and Penent F 2003 Phys. Rev. Lett. 90053003

Eland J H D 2009 Adv. Chem. Phys. 141 103-152

Hayaishi T, Murakami E, Yagishita A, Koike F, Morioka Y and Hansen J E 1988 J. Phys. B: At. Mol. Phys. 213203

Hayaishi T, Yagishita A, Shigemasa E, Murakami E and Morioka Y 1990 J. Phys. B: At. Mol. Phys. 234431

Hayaishi T, Murakami E, Morioka Y, Shigemasa E, Yagishita A and Koike F 1994 J. Phys. B: At. Mol. Opt. Phys. $27 \mathrm{~L} 115$

Hayaishi T, Tanaka T, Yoshii H, Murakami E, Shigemasa E, Yagishita A, Koike F and Morioka Y 1999 J. Phys. B: At. Mol. Opt. Phys. 321507

Hayaishi T, Fujita Y, Izumisawa M, Tanaka T, Murakami E, Shigemasa E, Yagishita A. and Morioka Y, 2000 J. Phys. B: At. Mol. Opt. Phys. 3337

Hayaishi T, Matsui T, Yoshii H, Higurashi A, Murakami E, Yagishita A, Aoto T, Onuma T and Morioka Y , 2002 J. Phys. B: At. Mol. Opt. Phys. 35141

Hikosaka Y, Aoto T, Lablanquie P, Penent F, Shigemasa E, and Ito K 2006 Phys. Rev. Lett. 97053003 
Hikosaka Y, Lablanquie P, Penent F, Kaneyasu T, Shigemasa E, Feifel R, Eland J H D and Ito K 2009 Phys. Rev. Lett. 102013002

Hikosaka Y, Lablanquie P, Penent F, Selles P, Kaneyasu T, Shigemasa E, Eland J H D and Ito K 2009A Phys. Rev. A 80 031404(R)

Ito K, Penent F, Hikosaka Y, Shigemasa E, Suzuki I H, Eland J H D, Lablanquie P 2009 Rev. Sci. Instrum. 80, 123101

Jurvansuu M, Kivimäki A and Aksela S 2001 Phys. Rev. A 64, 012502

King G, Tronc M, Read F and Bradford R 1977 J. Phys. B: At. Mol. Opt. Phys. 102477

Koike F, 1994 Phys. Lett. 193A 173

Kuchiev M Yu and Sheinerman S A 1986 Sov.Phys. - JETP 63986

Kuchiev M Yu and Sheinerman S A 1989 Sov. Phys.-Usp. 32569

Kjeldsen H, Thomas T D, Lablanquie P, Lavollée M, Eland J H D, Penent F, Hochlaf M and Hall R I, 1996 J. Phys. B: At. Mol. Opt. Phys. 291689

Lablanquie P, Sheinerman S, Penent F, Hall R I, Ahmad M, Hikosaka Y and Ito K, 2001 Phys. Rev. Lett. 87053001

Lablanquie P, Sheinerman S, Penent F, Hall R I, Ahmad M, Aoto T, Hikosaka Y and Ito K, 2002 J. Phys. B: At. Mol. Opt. Phys. 353265

Lablanquie P, Andric L, Palaudoux J, Becker U, Braune M, Viefhaus J, Eland J, and Penent F 2007 J. Electron Spectrosc. Related Phenom. 156-158 51

Matsui T, Yoshii H, Higurashi A, Murakami E, Aoto T, Onuma T, Morioka Y, Yagishita A and Hayaishi T, 2002 J. Phys. B: At. Mol. Opt. Phys. 353069

Palaudoux J, Lablanquie P, Andric L, Eland J H D and Penent F 2008 Journal of Physics: Conf.Ser. 141012012

Penent F, Palaudoux J, Lablanquie P, Andric L, Feifel R and Eland J H D 2005 Phys. Rev. Lett. 95 083002

Ralchenko, Yu., Kramida, A.E., Reader, J., and NIST ASD Team (2008). NIST Atomic Spectra Database (version 3.1.5), [Online]. Available: http://physics.nist.gov/asd3 [2009, December 11]. National Institute of Standards and Technology, Gaithersburg, MD.

Schmidt V, 1992 Rep. Prog. Phys. 55, 1483

Sheinerman S A, 1994 J. Phys. B: At. Mol. Opt. Phys. 27 L571

Sheinerman S A, 1998 J. Phys. B: At. Mol. Opt. Phys. 31 L361

Sheinerman S, Lablanquie P, Penent F, Palaudoux J, Eland J H D, Aoto T, Hikosaka Y and Ito K 2006 J. Phys. B: At. Mol. Opt. Phys. 391017

Shigemasa E, Toyoshima A, Yan Y, Hayaishi T, Soejima K, Kiyokura T, and Yagishita A, 1998 J. Synchrotron Radiat. 5777

Viefhaus J, Cvejanovic S, Langer B, Lischke T, Prumper G, Rolles D, Golovin A, Grum-Grzhimailo A, Kabachnik N, Becker U, 2004 Phys. Rev. L 692, 083001

\section{Figure Captions}

Figure 1: a) $\mathrm{Ar}^{2+}$ states populated by photons of $\omega=251.63 \mathrm{eV}$. The histogram was obtained from the energy sum of all two-electron pairs detected in coincidence. It is converted to binding energy of the final $\mathrm{Ar}^{2+}$ states by the relation : $\omega=$ $E_{B}\left(\mathrm{Ar}^{2+}\right)+E 1+E 2$. The discretisation step is $50 \mathrm{meV}$. Accumulation time was 4 hours and the number of electrons detected was kept constant at 3000/s by adjusting the width of the monochromator exit slit, in order to compensate for the decrease with time of the photon flux. b) $\mathrm{Ar}^{3+}$ states populated by photons of $\omega=251.63 \mathrm{eV}$. In the same way as for a), the histogram was obtained from the energy sum of all three electrons detected in coincidence. It is converted in binding energy of the final $A r^{3+}$ 
states by the relation : $\omega=E_{B}\left(\mathrm{Ar}^{3+}\right)+E 1+E 2+E 3$. Uncertainty in the energy scale are evaluated to be of the order of $0.3 \mathrm{eV}$.

Figure 2: a) Two dimensional coincidence map showing the energy correlations between three electrons detected in coincidence. The sum of the energies of the three electrons is imposed to lie between 160 and $170 \mathrm{eV}$ to ensure that a final $\operatorname{Ar}^{3+}\left(3 p^{-3}\right)$ is reached. Coincidence counts are represented as a function of two electrons of the triplet only, as the energy of the third electron is determined by the relation : $\omega=E_{B}\left(\mathrm{Ar}^{3+}\right)+E 1+E 2+E 3$. Intensity is encoded in color with a log scale. Green rectangles visualize the energy regions analyzed in Fig 3,5 and 6. b) displays the projection of the coincidence map onto the ordinate axis. It gives an image of the energy distribution of one of the electrons emitted by $\omega=251.63 \mathrm{eV}$ photons when producing $\mathrm{Ar}^{3+}\left(3 p^{-3}\right)$ states.

Figure 3: a) PCI distortion of the $\operatorname{Ar} 2 p$ photoelectron lines associated with a direct Double Auger decay populating $\mathrm{Ar}^{3+}\left(3 p^{-3}\right)$ final states. Photon energy was $\omega=251.63 \mathrm{eV}$. The kinetic energy of one of the Auger electron has been chosen in the (30- 70) eV range (blue) or in the $(4.9-5.1) \mathrm{eV}$ one (red). It is compared with the PCI distortion in a single Auger decay forming $\mathrm{Ar}^{2+}\left(3 p^{-2}\right)$ final states (in black). Dots with error bars (two standard deviations from the counting statistics) represent experimental results. Lines give the prediction from our eikonal model, after a $+140 \mathrm{meV}$ shift of the energy scale and their convolution with a Gaussian of FWHM $=50 \mathrm{meV}$ to account for the experimental resolution. b) Same but obtained at a photon energy $\omega=260.63 \mathrm{eV}$, $12 \mathrm{eV}$ above the $2 p_{3 / 2}$ level. Here, the shift of the calculated line shapes is $+90 \mathrm{meV}$ and convolution is done with a FWHM $=150 \mathrm{meV}$ Gaussian.

Figure 4: Non coincident high resolution electron spectra obtained with the MBS electrostatic analyzer at a photon energy of $270 \mathrm{eV}$. a) was obtained with a $2 \mathrm{eV}$ pass energy and is fitted by the sum of two $5.2 \mathrm{meV}$ FWHM Gaussians (red line). The component on the high energy side of the main peak is attributed to the contribution of a weak CDA path mediated by an $\mathrm{Ar}^{2+}$ intermediate different from A1. b) was obtained with a $20 \mathrm{eV}$ pass energy and is fitted by two Voigt profiles (in blue, with their sum represented by the red curve), corresponding to the decay of the A2 intermediate state to the $\operatorname{Ar}^{3+}\left(3 p^{-3}\left({ }^{2} P_{3 / 2}\right)\right)$ and $\left({ }^{2} P_{1 / 2}\right)$ final states.

Figure 5: a) In red: PCI distortion of the Ar $2 p$ photoelectron lines associated with the cascade Double Auger decay. Photon energy was $\omega=251.63 \mathrm{eV}$. A filter on the kinetic energy of the second Auger electron has been imposed, $(2.1-2.3) \mathrm{eV}$, so that we select the $\mathrm{Ar}^{2+*}$ excited state of $86.31 \mathrm{eV}$ binding energy decaying to $\operatorname{Ar}^{3+}\left(3 p^{-3}\left({ }^{4} S\right)\right)$. It is compared with the PCI distortion in single Auger (black) and double Auger decays (blue) from figure 3. Other conventions are the same as in Figure 3. b) Same but obtained at a photon energy $\omega=260.63 \mathrm{eV}$.

Figure 6: Same as for Fig 5, but the $(8.2$ - 8.7) eV filter on the Auger electron energy selects the $\mathrm{Ar}^{2+*}$ excited state of $96.9 \mathrm{eV}$ binding energy decaying to $\operatorname{Ar}^{3+}\left(3 p^{-3}\left({ }^{2} P\right)\right)$.

Figure 7: PCI shift of the $8.5 \mathrm{eV}$ Auger electron emitted in the A2 Cascade Double Auger path. The data comes from events where three electrons have been detected in 
coincidence and are associated to the formation of a final $\mathrm{Ar}^{3+}\left(3 p^{-3}\right)$ state. Blue, black and red curves correspond respectively to coincidences of the second step Auger electron with $\mathrm{a} \sim 12 \mathrm{eV} 2 p_{3 / 2} \mathrm{a} \sim 3 \mathrm{eV} 2 p_{3 / 2}$ photoelectron and a $\sim 0.9 \mathrm{eV} 2 p_{1 / 2}$ photoelectron, see text. 

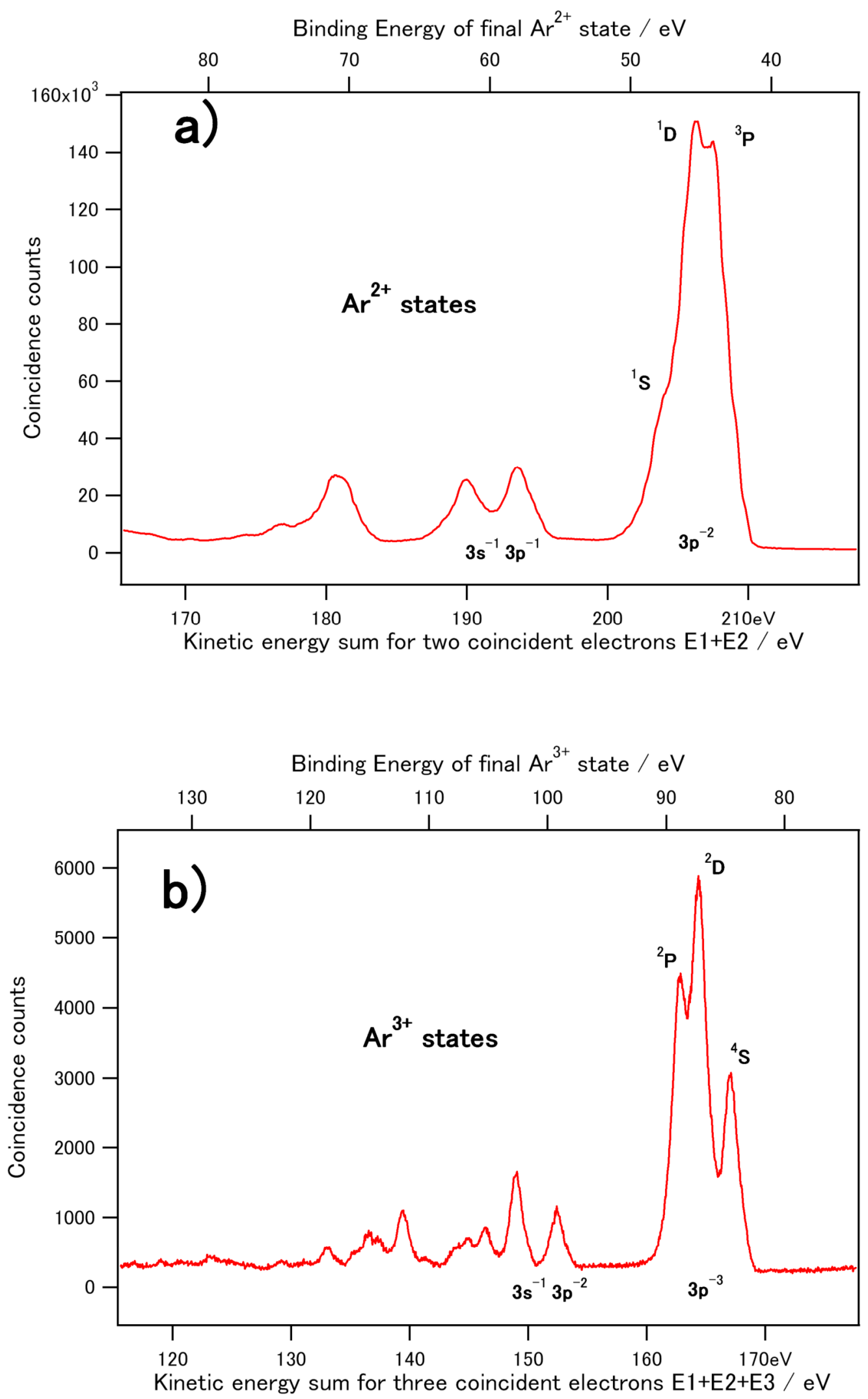

FIGURE 1 


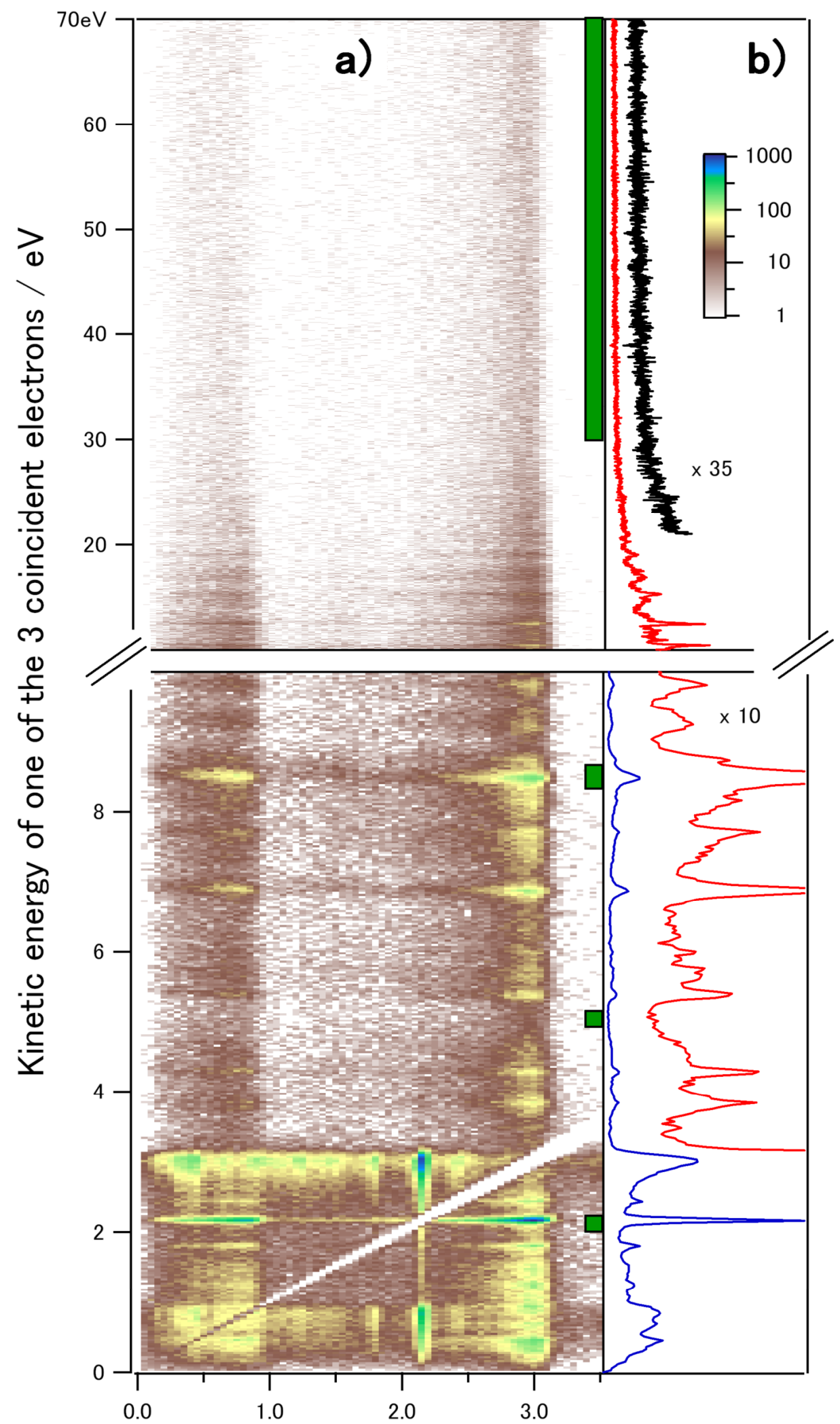

Kinetic energy of one of the 3 coincident electrons / eV

FIGURE 2 

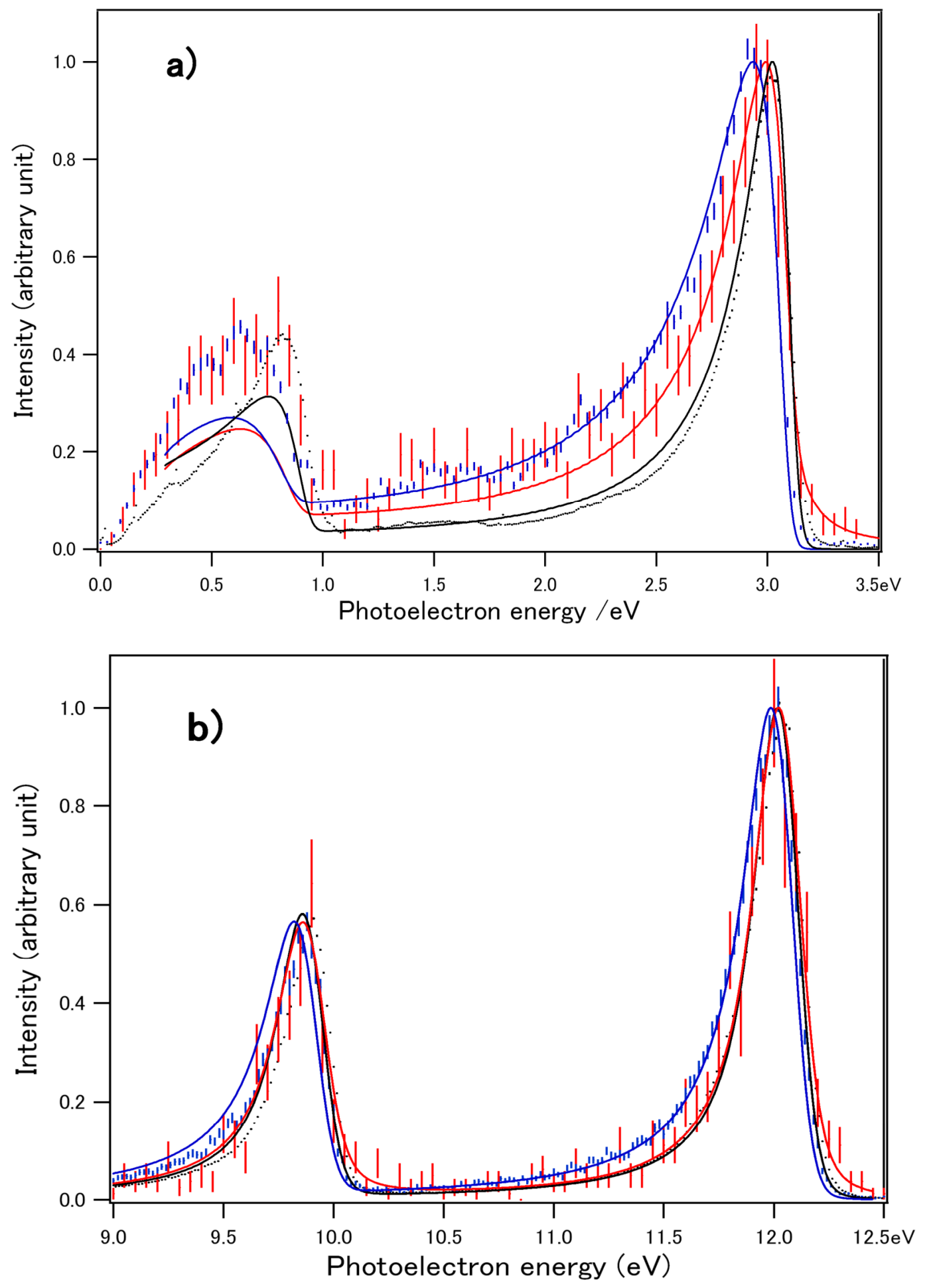

FIGURE 3 

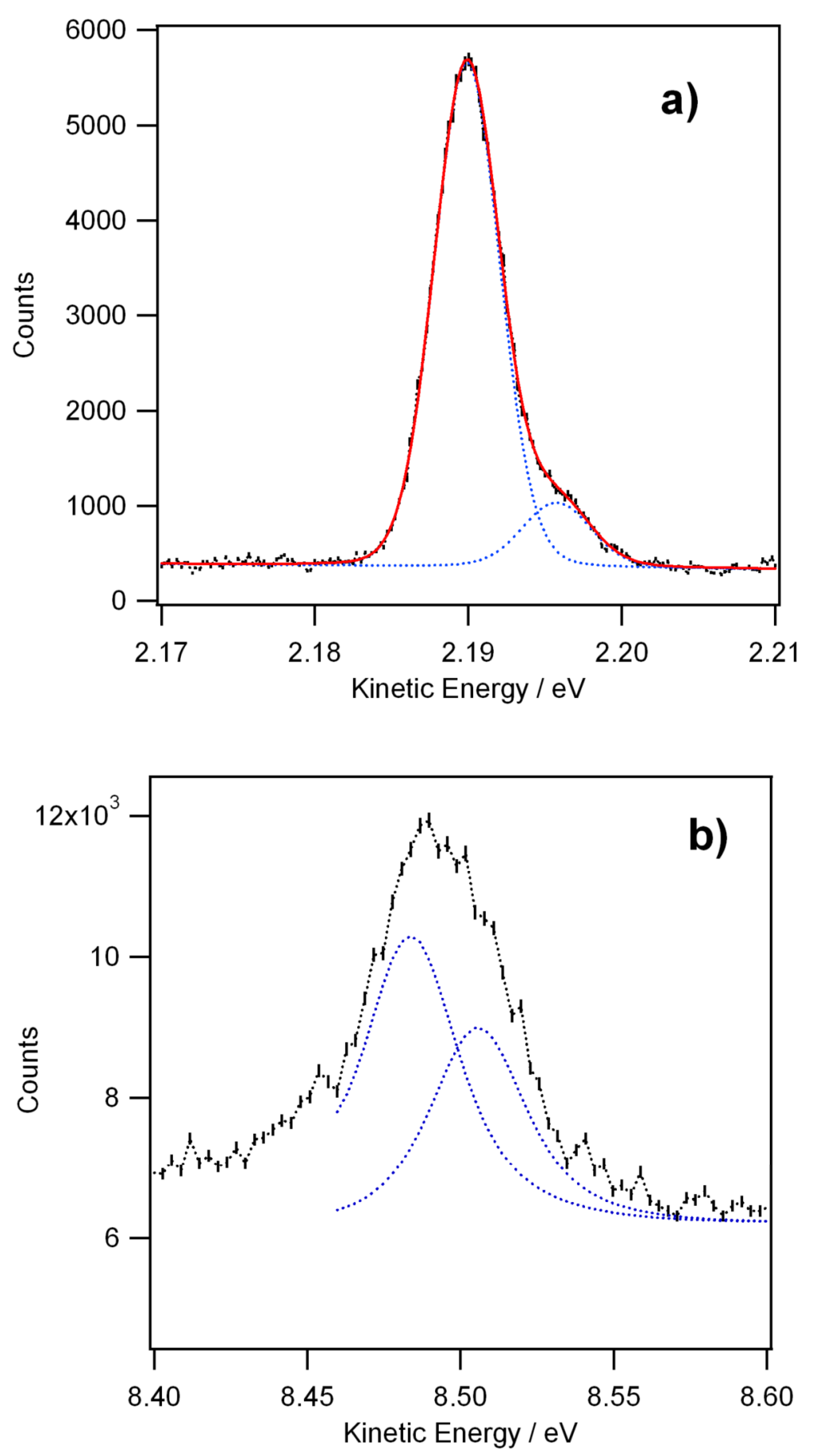

FIGURE 4 

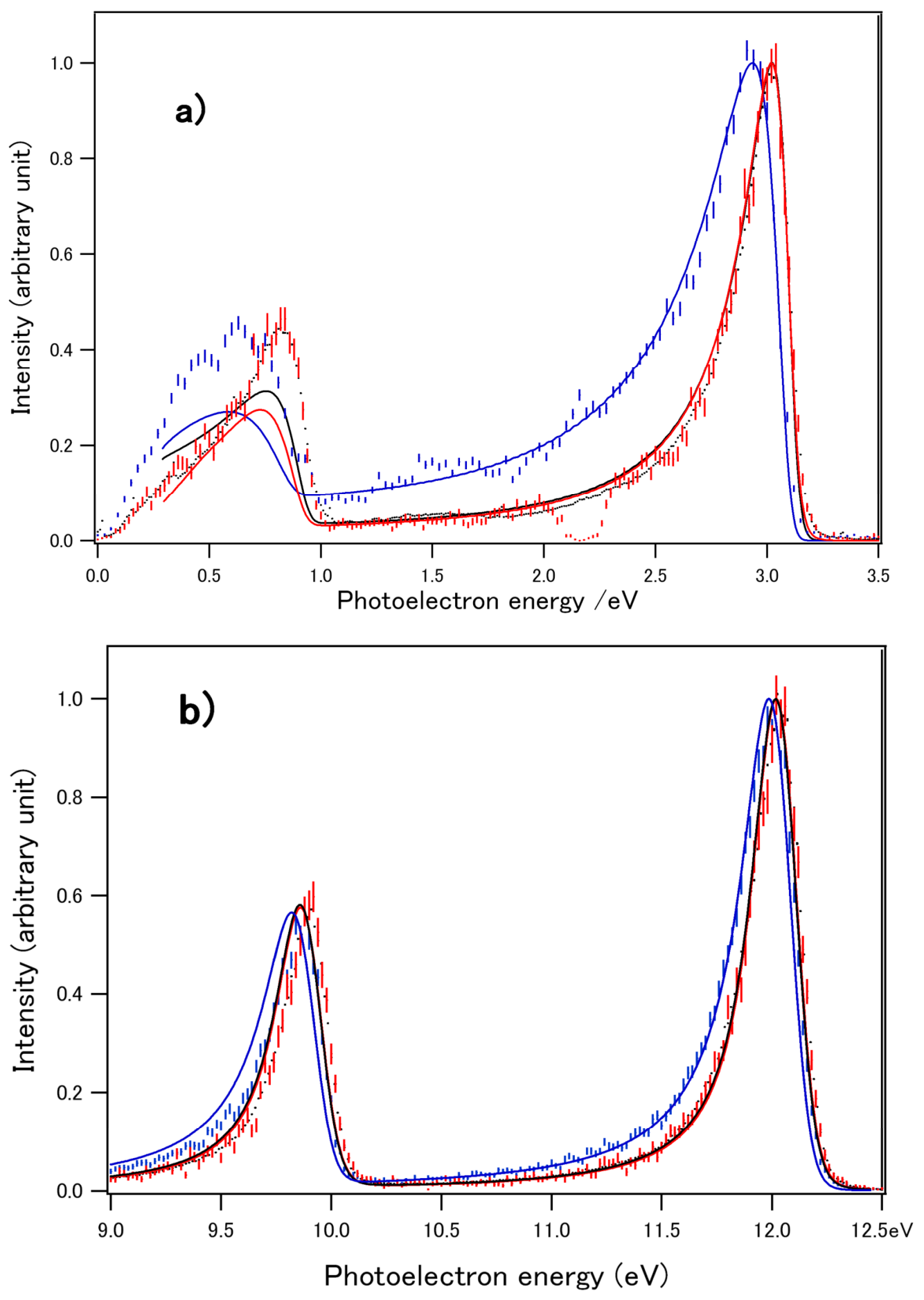

FIGURE 5 

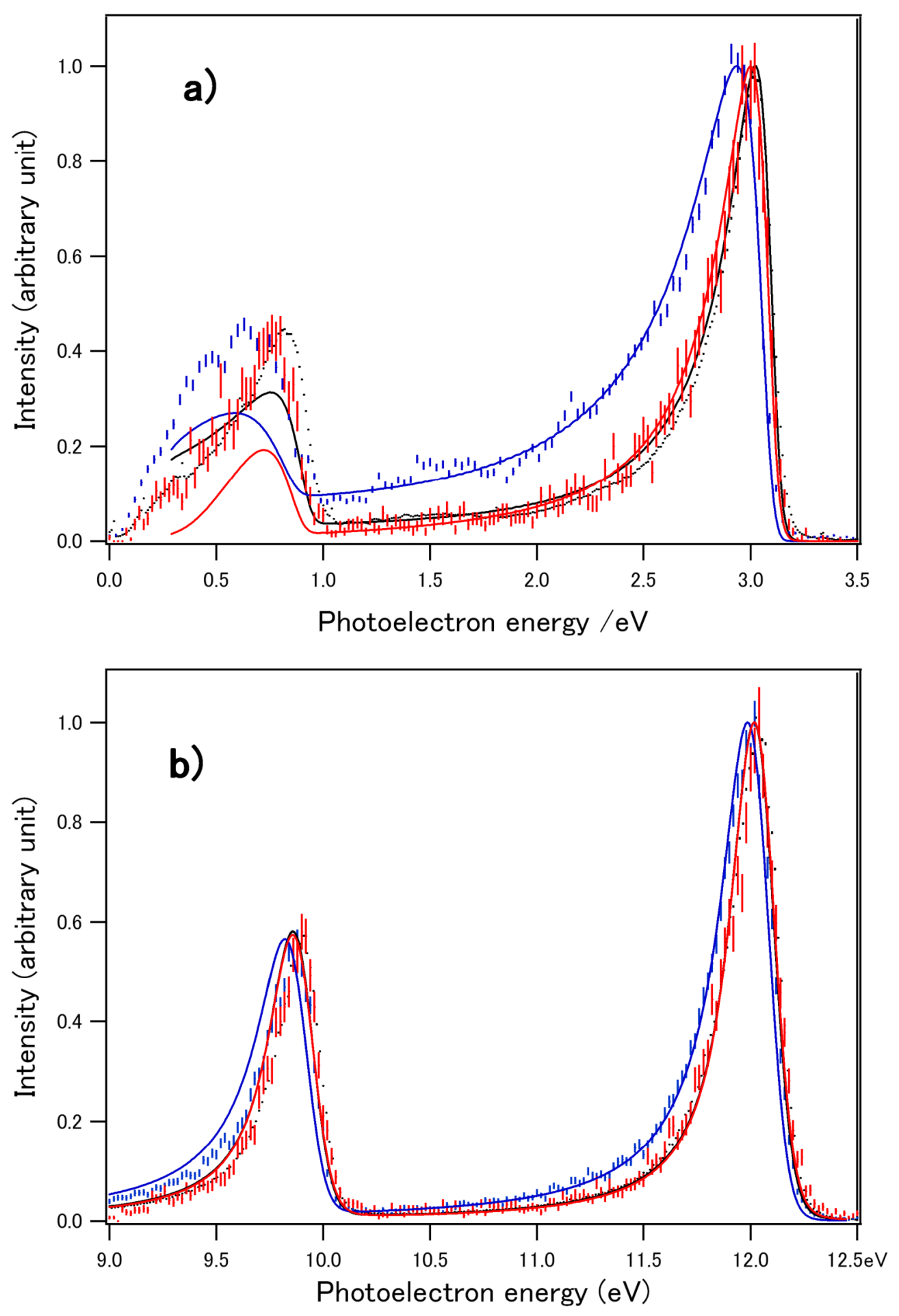

FIGURE 6 




FIGURE 7 